\title{
Profil et épaisseur des mots : Kamau Brathwaite, RêvHaïti
}

\author{
Christine PAGNOULLE
}

Kamau Brathwaite est un des ces écrivains dont l'écriture oblige à tracer de nouvelles pistes de pensée, dont les jeux — de registres, de typographies, de sonorités, de significations — ouvrent des perspectives neuves. Originaire de la Barbade, Brathwaite fait des études universitaires d'histoire en Grande-Bretagne, puis passe quelques années au Ghana. Il s'installe ensuite pour vingt-cinq ans en Jamaïque, où il est professeur d'histoire à l'Université des Indes Occidentales, Mona (Kingston). Avec sa femme Doris, qu'il appelait Mexican, il vivait dans un village des Blue Mountains derrière la capitale — Irish Town (IT). En 1986, sa femme meurt. En 1988, l'ouragan Gilbert détruit ses archives : les papiers et les ordinateurs. En 1990, il se fait agresser et dévaliser à Trench Town, quartier notoirement dangereux de Kingston. Comme il l'explique à Gordon Rohlehr, ces trois événements vont constituer une rupture, une faille "like a kind of RIFT Valley in my senscape" ${ }^{1}$. La coupure se marque dans sa vie. Il quitte la Jamaïque, où il se sentait de moins en moins compris ; il s'installe aux Etats-Unis, où il est maintenant professeur de littérature afro-caribéenne à New York University. Elle se marque aussi dans son écriture. D'une part, il n'y aura plus de distinction entre genres, plus de différence entre poésie et prose, plus besoin d'excuse pour des notes explicatives, qui sont prétextes à nouveau jeux de langue, comme dans le livresomme sur la littérature de la Barbade, Barabajan Poems. D'autre part, il se met à jouer, à créer, avec différentes polices, différentes tailles, différents styles. C'est le "videostyle" ou "style vidéo" dont il va être question plus loin.

Le texte auquel ces pages sont consacrées, DreamHaiti, publié par Savacou North en 1995 est aussi "DreamHaiti", une des sept "rêvhistoires" (DreamStories) publiées chez Longman en 1994. Il s'agit là de textes tout à fait inclassables dans les genres et catégories familiers, mais où, quelle que soit la mise en page, l'élément poétique (utilisation créatrice de la langue) est bien plus important que l'élément prosaïque (utilisation de la langue comme instrument d'information). Le ton et l'atmosphère changent d'une histoire à l'autre, allant du plus enfermé dans l' "i sol ence" tragique de la douleur ("4 $4^{\text {th }}$ Traveller") jusqu'à la voix collective, et clairement politique, qui s'exprime dans "DreamHaiti". Le contexte dans lequel le texte se situe est celui des années quatrevingt, quand la misère était si grande dans les dernières années du règne de Jean-François Duvalier que les Haïtiens, plus encore que les habitants d'autres îles caraïbes, n'espéraient qu'une chose: la chance de pouvoir quitter leur île, à peu près dans n'importe quelles conditions. D'où des images pathétiques et trop familières de "boat people" agglutinés sur des bateaux surchargés ${ }^{2}$.

A partir de la voix, de la musique des Caraïbes anglophones où il a vécu — la Barbade, la Jamaïque Brathwaite a créé sa propre langue, un jeu de sons et de sens dont il élabore les règles. Ceci justifie je l'espère en partie la présomption avec laquelle moi qui n'ai jamais vécu aux Antilles, qui ne connais le parler des îles caraïbes que par ce que je lis, ai entrepris la traduction de certaines de ses œuvres ${ }^{3}$.

Un simple coup d'œil au texte repris dans le fascicule de référence permet de comprendre un des sens des termes "profil" et "épaisseur" dans le titre. Mais s'il s'agira bien de déterminer le rôle de la typographie dans la portée du texte, je m'attacherai également à une autre lecture possible des mots "profil" et "épaisseur" : le jeu sur le double sens, les glissements et ouvertures qu'il provoque presque à chaque ligne.

Avant d'offrir quelques commentaires sur la typographie du texte, je voudrais attirer l'attention sur trois

\footnotetext{
${ }^{1}$ Introduction à DreamStories. Harlow: Longman, 1994 : p.iii.

${ }^{2}$ DreamStories est une des trois œuvres majeures publiées après la césure à la fin des années 80 . Un premier ouvrage de cette période avait été publié en 1993 par les presses de l'université du Wisconsin, The Zea Mexican Diary. C'est un poignant témoignage d'amour en même temps que l'itinéraire d'un deuil. L'anthologie Barabajan Poems a paru en 1995 chez Savacou North. Mon article "Kamau Brathwaite: A Voice Out of Bound", à paraître dans Union in Partition: Essays in Honour of Jeanne Delbaere, ed. Gilbert Debusscher \& Marc Maufort, Liège: $\mathrm{L}^{3}, 1997$ présente la voix nouvelle de Brathwaite dans ces derniers ouvrages en date.

${ }^{3}$ Outre le texte présenté ici et un poème tiré du recueil X/Self (Oxford: OUP, 1987), "Mont Blanc", publié dans la revue $\pi$ Magazine de poésie, j'avais traduit quatre poèmes, avec Maria Francesca Mollira, publiés sous le titre collectif The Visibility Trigger/LeDétonateur de visibilité (Leuven: Leuvenses Schrijversaktie,1986).
} 
différences essentielles entre la présentation du texte reproduit, qui est celui de Savacou North ${ }^{4}$ et celui publié chez Longman.

- Le texte Longman présente nettement moins de divergences par rapport aux normes grammaticales et orthographiques de l'anglais britannique que le texte Savacou.

— Certains passages figurent uniquement dans le texte Savacou ; ainsi, la partie du texte qui est pour moi la plus chargée d'émotion, après la référence à la poussière du désert d'Atacama, n'apparaît que dans le texte Savacou.

— Le nombre de changements de polices et l'espace occupé par des blancs sont beaucoup plus limités dans l'édition commerciale (Longman), de même qu'y ont été supprimés les très grands caractères (US Coast Guard Gutter), les caractères-images (comme la mitraillette dans le nom de lieu la Crête à Pierrot) et des irrégularités de typographie (comme la bousculade cahotante des lettres du long paragraphe page 47).

L'argument qui pèse le plus lourd contre l'utilisation de différentes polices est l'argument économique de l'éditeur : ce sont là des jeux qui coûtent cher. Indépendamment de considérations économiques, certains critiquent ces jeux typographiques comme étant purement formels, voire parfaitement gratuits. Mais l'objection est étrange vis-à-vis d'un texte littéraire, qui est, par essence même, forme gratuite. L'œuvre d'art est par définition intransitive, et n'existe que par sa forme, ce qui ne veut pas dire qu'elle existe en dehors du temps ou des contingences historiques, pour Brathwaite moins que pour personne d'autre. L'élément dérangeant ne peut pas être que la poésie se frotte au graphisme : ce n'est vraiment pas la première fois que cela arrive. Il suffit de penser à Apollinaire. Va-t-on objecter qu'il y a compromission entre poésie et technologie informatique ? Il y a en tout cas retournement. C'est, pour reprendre les mots de Brathwaite, Caliban "cursing Prospero with his own curser", la touche Retour qui revient dans les gencives de Prospero, la souris qui se met à grignoter les textes canoniques...

Quand il défend la technique de Brathwaite dans son introduction à DreamStories, Gordon Rohlehr écrit que le but est de déstabiliser le lecteur en prenant ses attentes à contre-pied ${ }^{5}$. C'est là effectivement un procédé assez fréquent chez Brathwaite ; mais, dans ma lecture, la fonction des changements de polices est presque l'inverse. C'est, d'une part, comme l'a écrit Brathwaite lui-même, une façon de faire voir ce que le poète entend dans sa tête, de donner forme sur la page aux intonations du texte. C'est aussi parfois une façon de donner une dimension supplémentaire aux mots.

Il me semble évident que malgré les difficultés techniques, la publication de la traduction comme de l'original doit s'efforcer de respecter la typographie originale.

Les quelques remarques qui suivent permettent d'aborder le thème du texte par le biais de la typographie. Les lettres allongées, un peu confuses, suggèrent à la fois la décomposition du corps dans l'eau et l'incertitude quant à l'identité du locuteur, qui est tour à tour ou simultanément le nègre noyé il y a bien longtemps lors d'un déracinement/arrachement en cale négrière, le poète égaré en rêve sur le pont d'une vedette garde-côtes US et le réfugié haïtien, parti dès avant l'aube de derrière le morne d'Estagnes sur les hauteurs de Port-au-Prince, maintenant noyé lui aussi comme cet enfant/bougre/bouée, cet "objet Sun Bryan", ce triangle repêché dans les eaux de l'infortune (et la forme triangulaire est probablement un rappel du trafic entre l'Europe, l'Afrique et le Nouveau Monde, une allusion à ce commerce de "bois d'ébène" qui a si largement contribué au développement économique de l'Europe occidentale).

L'expression "réfugiés haïtiens" est évidée, réduite à un bloc difficilement lisible et tenue à distance par les guillemets. Dans le texte Savacou, elle est de plus isolée sur une page pour elle toute seule, comme en quarantaine.

Staline le Noir, "Black Stalin" est lui aussi fondu en un bloc à peine déchiffrable, mais pour lui la masse est noire et pleine d'aspérités, rappelant peut-être l'état de guerre dans lequel l'URSS a été maintenue tout au long de ses quelque soixante-dix ans d'existence.

Certaines lettres sont transformées en images. Si le ê de La Crête est transformé en mitraillette (p. 47), c'est pour rappeler le sort des Noirs massacrés par les troupes coloniales de la France révolutionnaire à la fin du XVIIIe

\footnotetext{
${ }^{4}$ DreamHaiti. New York: Savacou North, 1995 (Les références de pages dans le texte sont à cette édition.)

$5 " .$. the aim being to unsettle the reader's expectations by an unfixing or unhinging of word and image", Introduction : p.viii.
} 
siècle et le massacre continué, direct ou indirect, de ceux qui tentent de survivre. Le soleil qui rayonne au milieu de "cité soleil" à la page suivante est là sur le mode ironique, puisque la promesse de lendemains radieux pour les masses oubliées de la population, formulée avec tant de force persuasive par le docteur et ministre François Duvalier en 1954, était aussi vide et vaine que l'espoir d'accoster sur une terre d'accueil et de justice qui nourrit les réfugiés sur leurs canots.

Comment faut-il lire la répétition des signes ${ }_{-}{ }_{-}$des pages 41 à 44 , à propos du corps retiré des flots et que le locuteur/observateur/poète va y laisser retomber ? Ils peuvent évoquer l'image du toit, mentionnée lorsque le poète se voit ballotté par les vagues de l'Atlantique (pp. 22-23) : "avec je et moi comme seule tête / dépassant du chaume des vagues". Mais il me semble plus probable que ce qui est évoqué ici, c'est le "nam", la forme secrète du nom ("name" sans la poignée du e final), le mot qu'il ne faut pas prononcer sous peine de perte de pouvoir, l'identité que les Noirs, à qui tout a été volé y compris leur nom propre, vont cacher avec soin. Le "nam" chez Brathwaite, c'est l'âme, le chi, la force qui habite chacun ; son intégrité réside dans la voyelle, le A (ici sans barre transversale), protégée par les deux consonnes nasales.

J'en viens à une observation qui amène une transition entre caractéristiques typographiques et problèmes de traduction proprement dits. L'inscription sur la bouée de sauvetage US Coast Guard Cutter est transformée par un simple allongement de la boucle du C en US Coast Guard Gutter. Modification lourde de sens d'un bateau à un égout. Pour l'inscription elle-même, comme il est assez invraisemblable que les bouées d'un bâtiment américain portent une inscription en français, j'ai pu sans remords garder les mots anglais. Dans le texte même, en revanche, il me fallait remplacer le jeu sur une seule lettre par un glissement sémantique. Je propose vedette/corvette/crevette, le dernier mot suggérant si l'on veut pourriture et décomposition. Nous sommes ainsi entrés dans l'épaisseur du double sens. Si la vue est largement sollicitée, l'ouïe contribue aussi, presque tout le temps, à créer le sens dans un jeu pluriel. Ici aussi, certains objectant que ces déplacements apportent peu à l'épaisseur du texte. Peut-être en effet Brathwaite se laisse-t-il parfois emporter par la facilité d'une confusion qui n'ajoute pas grand chose. Souvent, pourtant, il y a bien dimension supplémentaire.

Du point de vue du traducteur, le texte présente deux types de problèmes.

Certaines confusions existent en anglais et pas en français. C'est le cas pour "cutter" / "gutter" ou pour la prononciation identique de "boy" et de "buoy". Ici ma perplexité était totale, car je ne pouvais pas jeter la bouée par dessus bord. Je suggère "bouée" et "bougre", mais la similitude est très approximative et "bougre" ne convient pas partout. Heureusement le français offre d'autres confusions possibles, comme celle sur le sens de "gris". Il y aura donc effet de compensation, comme disent les manuels.

L'autre type de problèmes est lié aux transformations que Brathwaite fait subir aux mots qu'il emploie, et là le traducteur peut davantage espérer suivre le mouvement, en se rappelant que souvent sinon toujours c'est l'effet sonore qui est prioritaire par rapport au sens des mots pris isolément. C'est ainsi que s'il écrit (p. 43) "swinging slightly quiet/ ly // from like the old clotheshanger hunger of my hand", c'est l'écho que j'ai essayé de rendre, en remplaçant l'allitération en h par une assonance en in : "de comme un vieux cintre l'étreinte de ma main".

Si nous examinons le début du rêve-récit (qui n'est pas du tout le début du texte, j'y reviendrai), nous avons d'emblée la confusion de "slate grey" en "slake grey" ; je l'ai rendue par "gris armoise", "armoise" au lieu de "ardoise", famille de plantes parmi lesquelles l'absinthe. Le redoublement des membres démembrés dans "I memember" est moins convaincant dans ma traduction : "je me remécore" au lieu de "remémore". Ensuite il fallait rendre la rencontre du susurrement de l'eau sur les parois métalliques "comin in at this soft swishin diagonal diamond" ; là j'en ai peut-être un peu trop fait en traduisant "inlassables elles se rompaient contre ce diamant diagonal / en sibilations sybillines".

A la page 14, comment traduire "anyship anytime anytide" ? Je propose, consciente de la faiblesse de la suggestion : "ce n'était qu'un bâtiment un moment un jusant". Comment rendre avec une semblable économie le tangage évoqué dans les quatre syllabes "up/thin \& thick/down"? J'ai écrit "ce balancement lévé/leger \& lourd/crashé", mais le nombre de syllabes a augmenté. La suite du paragraphe joue sur la superposition d'expressions vantant un arrangement parfait et de références à des effondrements divers. J'ai choisi "réglissé" (réglé + glissé) pour "slipshape" au lieu de "shipshape". L'expression "Bristol fashion" était bien embarrassante à traduire ; un de ses sens est un doublet de "shipshape", mais par ailleurs elle renvoie à l'un des ports britanniques qui a le plus profité du commerce triangulaire. Il n'y a pas d'équivalent français qui combine les deux sens, mais je ne voulais pas perdre le second, j'ai donc utilisé le début d'une vieille chanson française "en revenant de la belle Rochelle". 
A la page 23 du texte anglais, "writhin" au lieu de "writin'" à la deuxième ligne du deuxième paragraphe suggère un entortillement confus que j'ai traduit par «escribouillant». Deux lignes plus loin, "supprisin" est un exemple de jeu peut-être pas pleinement convaincant, mais il me semblait que la surprise se faisait plus physique et j'ai traduit par "souprenant", comme quand on saisit quelqu'un par derrière sous les aisselles.

Le nom du canot surchargé de réfugiés est le "Salvages" (p. 24), que bien sûr je n'ai pas traduit. Mais le lecteur français entendra-t-il à la fois le sauvetage et les sauvages?

Dans ma traduction, le père (father) de l'expression modifiée "sea come no father" (further) devient terre: "Ici la mer s'atterre", ce qui l'arrête aussi. Mais l'on pourrait songer aussi à quelque chose comme "Ici la mer n'apert".

Dans le dernier quart du poème, là où la police Times Roman vient remplacer la police de caractères allongés et un peu tremblants dont je n'ai pas encore trouvé le nom et donc l'inscription, l'Histoire fait pleinement irruption dans le texte, avec "le bruit antique de l'eau qui s'engouffre dans nos vies". Page 39, nous retrouvons les grands noms de la révolution haïtienne : Toussaint Louverture, que Brathwaite nomme Toussaint Legba (du nom du dieu yoruba du seuil, de la transition) et son lieutenant Christophe, qui va s'auto-proclamer roi et faire construire une citadelle dans le nord de l'île, à La Ferrière, près de Cap Haïtien ${ }^{6}$.

Le texte imprimé en grands caractères et justifié à droite où Brathwaite s'indigne que l'on puisse lui reprocher son indignation (pp. 45-6) ne présente pas vraiment de difficulté de traduction, en tout cas dans la première partie, les mots "pogrom" et "programme" sont les mêmes en français et en anglais. Au passage d'une page à l'autre, l'effet est peut-être encore plus saisissant en français ("persis / tuante"). Enfin pour rendre "ghost words to holler this tale", avec l'écho du vide ("hollow") dans le hurlement, je propose "insouffler des mots pour éventer cette histoire".

J'ai signalé plus haut que la première page du récit-rêve n'était pas la première page du texte. Celui-ci en fait tire beaucoup de sa puissance de compassion du texte d'hommage en créole qui forme, avec sa traduction, comme une parenthèse dans laquelle s'inscrit la dérive du rêve. Compassion/empathie et dérive/lâcher prise/confiance dans nos capacités intuitives et associatives, ce sont là des notions qui président à tout travail de traduction, et particulièrement à la traduction d'un texte aussi délibérément élusif et associatif que celui-ci.

\footnotetext{
${ }^{6}$ L'expression "Napoleons joyless eyeless island of torture on the glacial seas of the Jura" est une façon quelque peu élaborée de mentionner le fort de Joux où Toussaint Louverture avait été emprisonné, et où il est mort de froid et de mauvais traitement.
} 